\title{
ECG screening in athletes: optional or mandatory?
}

\author{
E. E. van der Wall
}

Published online: 28 May 2015

(C) The Author(s) 2015. This article is published with open access at Springerlink.com

In April 2015, it was reported by the San Antonio ExpressNews (Texas, USA) that legislation, requiring that high school student athletes should undergo electrocardiographic (ECG) screening before participating in sports, passed with a clear majority through a second reading in the Texas House. At the same time, The Dallas Morning News pointed out that the American College of Cardiology (ACC) and the Texas Pediatric Society were against the measure.

There are few topics that lead to more controversy amongst physicians caring for young athletes than the correct method of pre-participation physical screening. On one hand, there is the conviction that physical screening has limited use if the end result is the final common pathway, that is unstable ventricular arrhythmias [1]; on the other hand, there is the belief that all young athletes, regardless of the sport and level of participation, require history, physical examination, (stress) ECG, echocardiography and, if necessary, even magnetic resonance imaging and computed tomography $[2,3]$.

What fuels such debates is the fact that sudden cardiac death (SCD) in the young athlete is estimated to occur in between 0.5 and 1 per 100,000 participating athletes below the age of 35 years [4]. In the USA, 50-75 deaths per year occur in young athletes; in France, this is approximately $10-15$ [5]. Although these are very low numbers, it should be realised that competitive young male athletes suffer a fivefold higher risk of sudden cardiac death than noncompetitive athletes, and male athletes aged more than 30

E. E. van der Wall $(\bowtie)$

Netherlands Society of Cardiology/Holland Heart House, Moreelsepark 1,

3511 EP Utrecht, The Netherlands

e-mail: eevanderwall@hotmail.com years bear a potential for arrhythmias, atrial fibrillation or a 20-fold higher possibility of SCD than female athletes. Ruwald et al. [6] showed recently in the European Heart Journal (EHJ, April 2015) that competitive sport is associated with a twofold increased risk of ventricular arrhythmias/death when compared with inactive patients and with patients who participated in recreational sport. When compared with inactive patients, recreational sport was not associated with an earlier onset of symptoms or increased risk of ventricular arrhythmias/death. Therefore, the question arises how to prevent these events in competitive sports, even if they are relatively rare. Do these events justify the use of all our medical armamentarium, in particular, an ECG or even an exercise ECG?

In Italy in 2006, Corrado et al. [7] were able to show a reduction in the incidence of sudden cardiac death due to the screening intervention and appropriate restriction of athletes with arrhythmogenic right ventricular dysplasia. Can these results be extrapolated to other countries [8]? Perhaps it is best to focus on some achievable goals in pre-participation screening: (1) identify individuals known to be at risk and allow stratification of these risks, and (2) make recommendations regarding participation.

In 2011, the Journal of the American College of Cardiology (JACC) featured an article entitled 'Mandatory Electrocardiographic Screening of Athletes to Reduce Their Risk for Sudden Death: Proven Fact or Wishful Thinking?', which suggests the latter [9]. The study analysed the incidence of sudden death among competitive athletes following the enactment of the 1997 USA National Sport Law, which mandates screening of all athletes with resting ECG and exercise testing. The average yearly incidence of sudden death or cardiac arrest events was 2.6 events per 100,000 athlete-years. The study concluded that mandatory ECG screening of athletes had no apparent effect on their 
risk of cardiac arrest. The authors suggested that the higher incidence of sudden death found in the Italian studies was not representative of the sudden death risk in the athlete population of most other countries and continents, which have a much lower incidence.

When considering the current guidelines and recommendations, there is a disparity between Europe and the USA regarding the advice one should follow. Both the ACC and the American Heart Association (AHA) still agree that the mandatory screening of all young athletes with an ECG is not warranted based on cost due to the large number of tests that would be required, the low incidence of sudden death among athletes in the USA as well as the concern for false-positive results. Proceedings of the ACC Sports and Exercise Cardiology Think Tank, Washington, DC, on 18 October 2012 (published in JACC, November 2014), reported that, before changes in screening programs are recommended in the USA, data should be acquired that can demonstrate an improved outcome in young, asymptomatic athletes and show that the benefit of screening outweighs the risks [10]. By contrast, both the European Society of Cardiology (ESC) and the International Olympic Committee (IOC) have an opposing view and recommend resting electrocardiograms for all young athletes before they are allowed to compete [11]. The ESC and IOC points of view appear to now be in line with the recent legislation bill in Texas, USA, requiring that high school students should undergo ECG screening before participating in sports.

This verdict is corroborated by a recent study from Leischik et al. [12] stating that there is a basic need for indicated examinations and/or preventive measures inside or outside of pre-competition screening, both for young and older athletes ( $<35$ and $>35$ years). Competitive ambitious athletes $<35$ years should undergo a specific cardiological examination including echocardiography and exercise test, and regular annual check-up examinations if problems occur and before starting a training session. This policy largely supports the Lausanne protocol (revised in 2013), followed by our national Society of Sports Medicine (VSG), advocating a 12-lead resting ECG in 'top sportsmen' $<35$ years of age (http://www.sportgeneeskunde.com/richtlijnen-vsg). Athletes $>30$ years of age, undergoing extreme endurance competition, for example, triathlon or marathon, should preferably be examined by stress echocardiography from a prognostic point of view [13]. According to the authors [12], the costs for screening examinations should be regarded as negligible, given the high expenditures for preparation and participation in marathon and triathlon competitions or intensive costs in professional football and other team sports. Prevention of sudden sport-related deaths does not only have an individual component, but also a significant social impact on physical activity in the general population. In this regard, further expenses, studies and well-founded pre-competition screening in the industrialised world are socially justified and financially reasonable. The authors clearly affirm that the long-lasting disputes about using resting-ECG in pre-competitive sport screening belong to the past and that exercise tests should not only be reserved for professional teams.

To conclude, recent strategies, in particular based on European studies, seem to be in favour of routinely performing ECG screening in all athletes involved in competitive sports activities, irrespective of age and (family) history. This policy has, of course, the necessary implications for the present pre-participation screening. To further improve pre-participation screening, a dedicated education and certification program on 12-lead ECG interpretation is needed $[14,15]$. Standardised screening tools that include screening and interpretation of both resting and exercise ECGs for targeted athlete populations at risk of SCD are required [16]. These recommendations are aimed to overcome the resistance raised by individuals and institutions to pre-participation screening with an ECG.

Open Access This article is distributed under the terms of the Creative Commons Attribution Noncommercial License which permits any noncommercial use, distribution, and reproduction in any medium, provided the original author(s) and source are credited.

\section{References}

1. Bredeweg SW, Takens LH, Nieuwland W. Periodical cardiovascular screening is mandatory for elite athletes. Neth Heart J. 2007; $15: 224-5$.

2. Inklaar H, Panhuyzen-Goedkoop NM. Periodical cardiovascular screening is mandatory for elite athletes. Neth Heart J. 2007; $15: 221-3$.

3. Braber TL, Mosterd A, Prakken NH, et al. Rationale and design of the Measuring Athlete's Risk of Cardiovascular events (MARC) study: the role of coronary $\mathrm{CT}$ in the cardiovascular evaluation of middle-aged sportsmen. Neth Heart J. 2015;23:133-8. doi:10.1007/s12471-014-0630-0.

4. Beus MF de. Sudden cardiac events in athletes. The SPORTCOR initiative [PhD thesis]. Utrecht (The Netherlands): University of Utrecht; 2010.

5. Marijon E, Tafflet M, Celermajer DS, et al. Sports-related sudden death in the general population. Circulation. 2011;124:672-81.

6. Ruwald AC, Marcus F, Estes NA 3rd, et al. Association of competitive and recreational sport participation with cardiac events in patients with arrhythmogenic right ventricular cardiomyopathy: results from the North American multidisciplinary study of arrhythmogenic right ventricular cardiomyopathy. Eur Heart J. 2015 April 20. pii:ehv110. Epub ahead of print.

7. Corrado D, Basso C, Pavei A, Michieli P, Schiavon M, Thiene G. Trends in sudden cardiovascular death in young competitive athletes after implementation of a preparticipation screening program. JAMA. 2006;296:1593-601.

8. Groeneweg JA, Heijden JF van der, Dooijes D, Veen TA van, Tintelen JP van, Hauer RA. Arrhythmogenic cardiomyopathy: diagnosis, genetic background, and risk management. Neth Heart J. 2014;22:316-25. doi:10.1007/s12471-014-0563-7. 
9. Steinvil A, Chundadze T, Zeltser D, et al. Mandatory electrocardiographic screening of athletes to reduce their risk for sudden death proven fact or wishful thinking? J Am Coll Cardiol. 2011;57:1291-6.

10. Lawless CE, Asplund C, Asif IM, et al. Protecting the heart of the American athlete: proceedings of the American College of Cardiology Sports and Exercise Cardiology Think Tank October 18, 2012, Washington, DC. J Am Coll Cardiol. 2014;64:2146-71. doi:10.1016/j.jacc.2014.08.027.

11. Corrado D, Pelliccia A, Bjornstad HH, et al. Cardiovascular preparticipation screening of young competitive athletes for prevention of sudden death: proposal for a common European protocol. Consensus statement of the Study Group of Sport Cardiology of the Working Group of Cardiac Rehabilitation and Exercise Physiology and the Working Group of Myocardial and Pericardial Diseases of the European Society of Cardiology. Eur Heart J. 2005;26:516-24.
12. Leischik R, Dworrak B, Foshag P, et al. Pre-participation and follow-up screening of athletes for endurance sport. J Clin Med Res. 2015;7:385-92. doi:10.14740/jocmr2129w.

13. Wall EE van der. Long-distance running: running for a long life? Neth Heart J. 2014;22:89-90. doi:10.1007/s12471-014-0521-4.

14. Pickham D, Chan G, Carey M. Pre-participation screening for athletes and the role of advanced practice providers. J Electrocardiol. 2015;48:339-344. doi:10.1016/j.jelectrocard.2015.03.003.

15. Heidbuchel H, Papadakis M, Panhuyzen-Goedkoop N, et al. Position paper: proposal for a core curriculum for a European Sports Cardiology qualification. Eur J Prev Cardiol. 2013;20:889-903.

16. Sande DA van de, Hoogeveen A, Hoogsteen J, Kemps HM. The diagnostic accuracy of exercise electrocardiography in asymptomatic recreational and competitive athletes. Scand J Med Sci Sports. 2015 Feb 3. doi:10.1111/sms.12420. Epub ahead of print. 